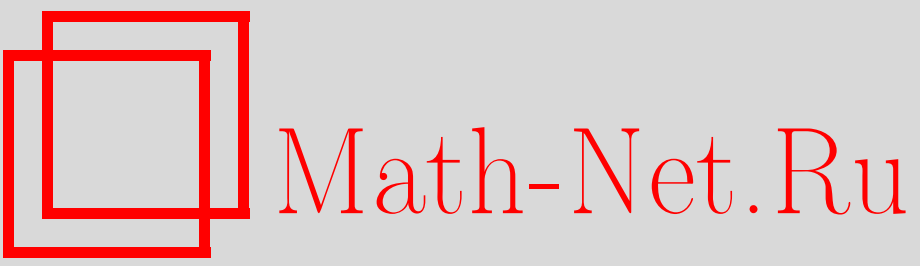

В. А. Зорич, Квазиконформные вложения римановых многообразий и теорема пикаровского типа, УМН, 1998, том 53, выпуск 1, 215-216

DOI: https://doi.org/10.4213/rm31

Использование Общероссийского математического портала Math-Net.Ru подразумевает, что вы прочитали и согласны с пользовательским соглашением

http://www.mathnet.ru/rus/agreement

Параметры загрузки:

IP : 34.229 .108 .108

26 апреля 2023 г., 16:55:36 


\title{
КВАЗИКОНФОРМНЫЕ ВЛОЖЕНИЯ РИМАНОВЫХ МНОГООБРАЗИЙ И ТЕОРЕМА ПИКАРОВСКОГО ТИПА
}

\author{
В. А. Зорич
}

Цель этой работы - теорема о продолжении ростка локально обратного отображения, которая могла бы служить инструментом исследования особенностей квазиконформных вложений римановых многообразий размерности $n \geqslant 3$.

Мы рассматриваем многообразия одной и той же размерности и, говоря о вложении, имеем в виду локально инъективное (локально обратимое) отображение. Квазиконформность вложения подразумевает ограниченность его коэффициента квазиконформности (по крайней мере в областях, где отображение инъективно).

Теорема 1 (основная теорема - альтернатива). Пусть $M^{n}$ u $N^{n}$ - римановы многообразия конечной размерности $n ; \dot{U}=U \backslash\{o\}$ - проколотая окрестность точки о $\in M^{n}$; $f: \dot{U}^{n} \rightarrow N^{n}$ - квазиконформное вложсение. Если $n \geqslant 3$, то справедлива следующая альтернатива:

(a) либо отображение $f$ инвективно в некоторой проколотой окрестности точжи $о$;

(b) либо любой росток $f_{p}^{-1}$ локально обратного отображения продолжается вдоль любого пути на $N^{n}$ с началом в точке $p$, не задевающего образ $f(\partial U)$ края окрестности $U$.

Следствием приведенной альтернативы, например, является теорема о глобальном гомеоморфизме (утверждающая, что при $n \geqslant 3$ всякое квазиконформное влоэсение $f: \mathbb{R}^{n} \rightarrow \mathbb{R}^{n}$ глобально обратимо) (см. [1], [2]), а также геометрическая версия этой теоремы, предложенная М. Громовым (см. [3], [4]).

В качестве попутного продукта получается также следующая

ТЕОРема 2 (пикаровского типа для квазиконформных вложений). Квазиконформное вложение одного риманова многообразия в другое риманово многообразие той же размерности $n \geqslant 3$

(а) либо инвективно в некоторой проколотой окрестности изолированной особой точки;

(b) либо образ любой такой окрестности покрывает все многообразие образа.

Отображение $z \mapsto \exp \frac{1}{z}$ показывает, что условие $n \geqslant 3$ здесь существенно.

Вместе с тем, обе возможности этой альтернативы реализуемы в любой размерности $n \geqslant 2$. Примером реализации может служить отображение Хопфа $f: \mathbb{R}^{n}=\mathbb{R}^{n} \backslash\{o\} \rightarrow S^{n-1} \times S^{1}$, определяемое соответствием $\mathbb{R}^{n} \ni x \mapsto\left\{2^{k} x \mid k \in \mathbb{Z}\right\} \in \dot{\mathbb{R}}^{n} / G$, где $G$ - группа гомотетий $g: \dot{\mathbb{R}}^{n} \rightarrow \dot{\mathbb{R}}^{n}$, порожденная отображением $x \mapsto 2 x$.

Доказательство основной теоремы опирается на ряд лемм. Ключевым является следующее вспомогательное утверждение о связи конформной емкости и геометрии видимой части границы звездной области.

ЛЕмма 1 (о звезде). Пусть $D \subset \mathbb{R}^{n}(n \geqslant 2)$ - область, звездная относительно точ$\kappa и ~ о \in \mathbb{R}^{n}$, т.е. $D$ образована бесконечными лучами или их конечными интервалами, исходящими из точки о и заканчивающимися (если интервал конечен) в точках (видимой из о) части $\Delta$ границы $\partial D$ области $D$. Пусть $\Gamma$ - семейство всех кривых в $D$, соединяющих в-окрестность $B($ о, $\varepsilon)$ точки о с $\Delta$.

Работа вьполнена при частичной поддержке Российского фонда фундаментальных исследований (грант № 96-01-01218). 
Если конформный модуль семейства Г равен нулю, то

- чентральная проекция $\Delta$ на сферу $\partial B($ о, $)$ не содержит отличных от точки связных компонент и

-

Напомним, что конформный модуль произвольного семейства $\Gamma$ кривых в $\mathbb{R}^{n}$ определяется равенством

$$
\bmod \Gamma:=\inf \int_{\mathbb{R}^{n}} \rho^{n} d v
$$

где нижняя грань берется по всем неотрицательным измеримым по Борелю функциям $\rho: \mathbb{R}^{n} \rightarrow \mathbb{R}_{+}$таким, что $\int_{\gamma} \rho \geqslant 1$ для каждой кривой $\gamma \in \Gamma$.

В доказательстве теоремы 1 , наряду с условием $n \geqslant 3$, существенно только то, что выходящее на особенность $о$ семейство кривых имело равный нулю конформный модуль и что образ этого семейства при вложении $f$ тоже имел конформный модуль, равный нулю.

Эти обстоятельства, с одной стороны, допускают расширение самого класса вложений $f$, например, можно считать $f$ квазиконформньм в среднем, а с другой стороны, позволяют сформулировать основную теорему в следующем более общем виде.

ТЕоРема 3 (о квазиконформном вложении многообразий конформно параболического типа). Пусть $U^{n}$ - п-мерное риманово многообразие с компактным (возможнно пустым) краем $\partial U^{n}$. Пусть $U^{n}$ имеет конформно параболический тип и пусть $f: U^{n} \rightarrow N^{n}-$ квазиконформное вложение $U^{n}$ в $n$-мерное риманово многообразие $N^{n}$.

Если $n \geqslant 3$, то любой росток $f_{p}^{-1}$ локально обратного отображения продолжается вдоль любого пути на $N^{n}$ с началом в точке $p$, не задевающего образ $f\left(\partial U^{n}\right)$ края u, бить может, еще некоторое (возможно, пустое) множество $S$ особенностей конформной емкости (и метрической размерности) нуль.

О конформном типе (в частности, о конформно параболическом типе) риманова многообразия см. статью [5].

\section{СПИСОК ЛИТЕРАТУРЫ}

[1] Зорич В. А. // Матем. сб. 1967. T. 74. С. 417-433. [2] Zorich V. A. // Lect. Notes in Math. 1992. V. 1508. P. 131-148. [3] Gromov M. // Proceedings of the 1978 Stony Brook Conference. Ann. of Math. Stud. V. 97, 1981. P. 183-213. [4] Gromov M. // Structures métriques pour les variétés riemanniennes. Notes du cours rédigé par J. Lafontaine et P. Pansu. Paris: CEDIC/Fernand Nathan et Soc. Math. de France, 1981. [5] Зорич B. А., Кесельман В. М. // Функцион. анализ и прилож. 1996. Т. 30. № 2. С. 40-55. 